\section{Restorative nomenclature}

Sir, we have noted that there seems to be a variation in the use of nomenclature when being applied to certain facets of restorative dentistry. One can usually communicate satisfactorily with professional colleagues when different terms are used for the same disease or treatment modality. Nevertheless, it is our opinion that we, as a profession, should be using the nationally-accepted nomenclature when describing certain aspects of restorative dentistry to facilitate communication. This is better achieved if one "vocabulary' is used.

In particular, alternative terms are often used when describing forms of removable and fixed prosthodontic treatment modalities, such as types of crowns or dentures. There are, of course, accepted guidelines as to the nomenclature that is accepted in the United Kingdom for many of these terms - one good example is the Prosthetic Dentistry Glossary, produced by the British Society for the Study of Prosthetic Dentistry. Other instances of common misuse of nomenclature are the uses of the terms 'chronic adult periodontitis' (as opposed to 'chronic periodontitis') and 'early onset' periodontitis (as opposed to 'aggressive periodontitis'); this, despite the fact that the accepted nomenclature of periodontal diseases was established several years ago (International Workshop for a Classification of Periodontal Diseases and Conditions. Annals of Periodontology 1999; 4: 1-112).
A. J. Preston
G. Kaur
By email

DOI: 10.1038/bdj.2007.405

\section{Unreasonable CPD}

Sir, I wholeheartedly agree with the sentiments expressed by T. Black regarding the online $\mathrm{CPD}$ articles (BDJ 2007; 202: 238).

I too prefer to read from a printed journal rather than a computer screen as:

1. It is much quicker to open a journal than go through the process of booting up a computer, logging onto the internet, accessing the website, entering passwords etc

2. Journals can be read anywhere - the same cannot be said for computers

3. Relevant CPD articles can also be saved in a file for future reference.

It is unreasonable to expect BDA members to download and print off articles when we pay such a high membership fee.

So please, please Mr Hancocks print both CPD articles!

\section{F. Sutton}

Bristol

DOI: 10.1038/bdj.2007.406

\section{Maintaining values}

Sir, I was interested to see and hear the media comment on Gordon Brown's recent dental treatment. Has there been a change in the way the profession now regards the issue of patient confidentiality, or has the naming of dentists and their patients now become an accepted part of our media dominated world?

Surely traditional values should be maintained in both our own and our patients' interests.

\section{S. Cripps}

Beaconsfield

DOI: 10.1038/bdj.2007.407

\section{Too late?}

Sir, I would like to congratulate you on your recent editorial A profession no longer (BDJ 2007; 202: 235). The proposal to 'stand down' the GDC is only the end of a long line of events to deprofessionalise dentistry.

Unfortunately much of the blame must lie with the GDC. Practitioners may now place full page advertisements in the press often making extravagant claims to expertise. Allowing them to practise in 'Health Boutiques' with beauticians and hairdressers, and to supplement their registered qualifications with unrecognised qualifications of doubtful validity, have all been contributory. These would have resulted in at least a disciplinary hearing if not erasure had the GDC not abrogated their responsibility.
When considered in relation to the clinical methods available at the start of the NHS, the complexity of our dental procedures and potential harm that is possible today means that the GDC should have shown itself determined to take strict control of the profession. I fear that it is now too late.

B. Scheer

London

DOI: $10.1038 /$ bdj.2007.408

\section{Keep it simple}

Sir, instead of Ken Eaton's intellectual gyration around the current rules, directives and interpretations of dental qualifications (Registration and the early years - the bad and the ugly BDJ 2007; 202: 173), would it not be simpler for everyone if the 'powers that be' wrote them simply, clearly and with consistency in the first place?

C. Debenham

London

DOI: 10.1038/bdj.2007.409

\section{Fluoride allergy}

Sir, this morning I had a new patient who presented having not been to the dentist for a long time. He explained that he had 'very bad teeth' and wanted to get them sorted out, but one of the main reasons for his bad teeth was his allergy to fluoride. A little cynical at first, I spent a while talking to him about this allergy. As a child he had a history of multiple collapses and after extensive testing it was decided that he had a severe allergy to the fluoride in his toothpaste. He has since avoided it at all costs and as a result is only able to clean his teeth with salt and some mouthwashes. When buying new oral hygiene products to try he is advised to send them for testing to check that they are fluoride free.

Clinically he presents with very few teeth and those that are left are very heavily restored. Interestingly enough radiographically there was no new caries.

According to the patient there are only around seven other such individuals in the UK who have this allergy to 
fluoride. My question to other practitioners is has anyone else come across this condition? Are there any suitable guidelines/products that anyone could recommend?

H. Keanie

Guildford

DOI: 10.1038/bdj.2007.410

\section{Therapy myths}

Sir, I am a dental therapist working in a busy Dental Access Centre. It was with despair that I received a questionnaire from a student therapist asking how many 'simple' fillings I complete in a day's work. While I acknowledge that the word 'simple' has been used in various documentation, I feel this one word has caused many members of the dental team to wrongly presume that therapists only carry out small occlusal restorations, and serves to perpetuate the myth that therapists only treat children.

The current situation is that therapists may carry out exactly the same cavity preparation and restoration, on exactly the same teeth, on exactly the same patients as a dental surgeon. What therapists may not undertake on these teeth are onlays, inlays, root canal treatments and the placement of amalgam retention pins (not that this would be a disadvantage to a therapist when there are good amalgam bonding agents available that negate the need to use pins).

A typical day sees me completing a variety of large multi-surfaced resin bonded amalgams on posterior teeth, and extensive composite restorations of carious/broken down anterior teeth, mainly on adult patients who have been unable to access dental care for a variety of reasons. In addition to this I carry out the full range of a therapist's duties which includes taking radiographs, administering block and infiltration local anaesthesia, extracting deciduous teeth etc.

A dental therapist does not need a dental surgeon on the premises while they are working; they must, however, have a written treatment plan for each patient that has been referred to them by a dental surgeon.

Dental therapists have been around for 45 years now; it is time for the dental profession to have a full understanding of what therapists may do, and appreciate what a valuable, highly skilled, and economical member of the team they can be.

J. Stockley

Dental Therapist

By email

DOI: 10.1038/bdj.2007.411

\section{Learn these lessons}

Sir, I read with much interest the paper by Patel and Bachelor outlining the experiences of vocational trainees $(B D J$ 2007; 202: 345-349). This supports the debate regarding modernising the application process to vocational training which has been ongoing for a number of years.

The date of publication of regional lists for vocational training has long been an issue of concern and has been repeatedly highlighted by the BDA Students' Committee and the British Dental Students Association (BDSA). To date, there has been no satisfactory reason why the regional deaneries are unwilling to harmonise the release dates, despite overwhelming pressure to do so.

However, I would raise a note of caution in driving change. With Modernising Dental Careers (MDC) looming it may well be timely to look at the results of Modernising Medical Careers (MMC) and the changes that have resulted for our medical colleagues.

A key change within MMC has been the introduction of the Medical Training Application Service (MTAS) to 'deliver a modernised and focused career structure' and 'streamline training'.

However, MTAS has failed spectacularly and has resulted in confusion and anxiety for medical trainees across the country. Professor Ian Gilmore (President of the Royal College of Physicians) has described the system as "so seriously flawed that it could not be relied on to select candidates for interview fairly'. The result has been thousands of our medical colleagues left with no prospect of interviews and 12,000 doctors taking to the streets of London and Glasgow, the biggest medical march in UK history. On Tuesday 6 March the Department of Health announced an 'independent' review of MMC and MTAS but has failed to achieve a solution and the interim proposals have resulted in the British Medical Association abandoning talks.

This cannot be allowed to happen within dentistry and we need to act now to prevent it. If not, MDC will be imposed upon us. We as a profession owe it to our trainees to be actively involved in talks, to see future proposals and comment upon them. To date, the Royal Colleges and Deaneries have failed to do so and are scrambling to rescue their trainees after the event. It's an outrageous position to be in.

Junior doctors have been treated appallingly. They have been let down by the Government, the Department of Health, those at MTAS and the Royal
Colleges. We need to learn these lessons and protect our dental graduates, our training and ultimately the safety of our patients.

\section{S. Rice}

London

DOI: 10.1038/bdj.2007.412

\section{Double teeth}

Sir, we would like to share with your readers an unusual case of a patient with double teeth. The 18-year-old male presented with inflammatory episodes in the right molar area, in relation to the partially erupted right third molar. Clinical and radiographic examination revealed gemination of the right third mandibular molar (Fig. 1). This tooth was removed entirely under local anaesthesia without odontosection (Fig. 2).

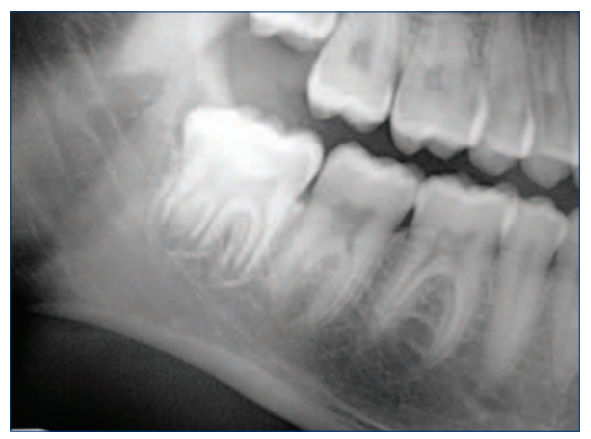

Fig. 1 Orthopantomograph showing 'double teeth' mandibular right third molar

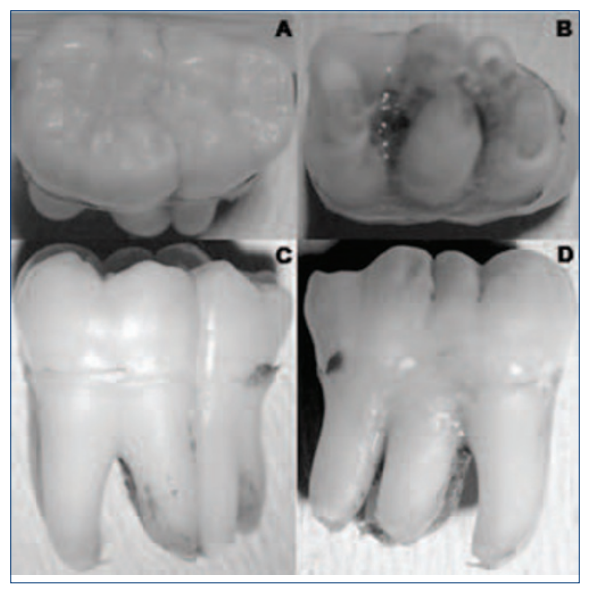

Fig. 2 Clinic image of tooth after exodontia. A: occlusal view. B: apical view. C: lingual view. D: buccal view

The anomaly of conjoined teeth has been described by different terms, such as gemination, fusion, double teeth and twining. These definitions of fusion or gemination are based on the way the tooth was developed. Gemination occurs as a result of attempted division of a single tooth germ, whereas fusion arises through the union of two normally separated tooth germs. Differential diagnosis is difficult; therefore several authors use the term 'double teeth'. 
This pathology usually involves anterior teeth, and is uncommon in premolars and permanent molars. ${ }^{1}$

The aetiology of gemination remains uncertain. Environmental factors, traumatisms, systemic diseases, vitamin deficits or genetic predisposition have been proposed for its development. ${ }^{2,3}$

Double teeth are generally asymptomatic. However, teeth may cause clinical and aesthetic problems, loss of the arch length, delayed or ectopic permanent teeth eruption, and periodontal diseases. ${ }^{4}$

Treatment is different between deciduous and definitive dentition. Exodontia is indicated when the anomalous deciduous tooth causes a permanent tooth altered eruption. Treatment in definitive double teeth depends on type, position in the dental arch and possible alterations affecting adjacent teeth. Exodontia, endodontics and aesthetics can be indicated. ${ }^{1-3}$ Surgical removal of the tooth and the paradental cyst is considered the treatment of choice when the involved tooth is a third molar. In our case, tooth extraction was indicated because of a lack of space and pericoronaritis episodes.

\section{P. Méndez \\ L. Junquera \\ L. Gallego \\ Spain}

1. Olivan-Rosas G, López-Jiménez J, Giménez-Prats M $\mathrm{J}$ et al. Considerations and differences in the treatment of a fused tooth. Med Oral 2004; 9: 224-228.

2. Chen HS, Huang Y L. Fusion of third and fourth mandibular molars? Oral Surg Oral Med Oral Pathol 1992; 73: 767.

3. Tomizawa M, Shimizu A, Hayashi S et al. Bilateral maxillary fused primary incisors accompained by succedaneous supernumerary teeth: report of a case. Int J Paediatr Dent 2002; 12: 223-227.

4. Duncan W K. Helpin M L. Bilateral fusion and gemination: a literature analysis and case report. Oral Surg Oral Med Oral Pathol 1987; 64: 82-87

DOI: 10.1038/bdj.2007.413

\section{Inadequate education}

Sir, doctors and medical students have been shown to be inadequately educated about oral diseases. ${ }^{1}$ This has dire consequences, not only for their patients but also for themselves. We report a case of a final year medical student who complained of a dull, aching pain on the lower right quadrant of the mandible. The pain was initiated by hot, cold and sweet stimuli. He ignored the advice of his fellow colleagues to seek dental treatment and subsequently self-medicated with a generic sodium fluoride mouthwash and ibuprofen. Pain relief was achieved, albeit temporarily which resulted in him bragging that a dental appointment was unnecessary. However, four weeks later, he developed

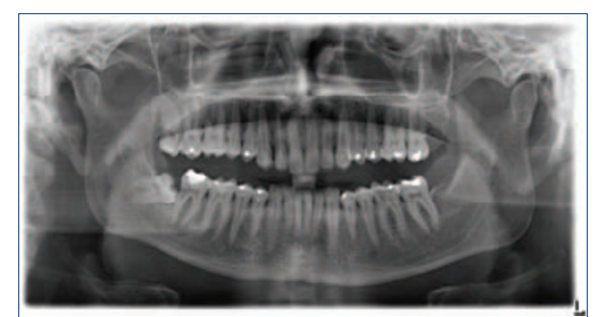

Fig. 1 Carious lesion on the distal of the LR7 which led to the teeth having a periapical infection

an abscess at the right angle of the mandible. Drainage was carried out by an emergency dentist through the soft tissues. An OPT was taken (Fig. 1) which showed a carious lesion on the distal of the LR7 which led to the teeth having a periapical infection. Further findings on the OPT showed a necrotic pulp on the LL6 showing an apical radiolucency on the distal root. The medical student is currently undergoing root canal treatment and has abandoned his treatment regime.

\section{A. Chai \\ D. S. Y. Chan \\ S. Malik \\ Cardiff}

Competing interests: S. M. was the unfortunate medical student.

1. McCann P J, Sweeney M P, Gibson J, Bagg J. Training in oral disease, diagnosis and treatment for medical students and doctors in the United Kingdom. Br J Oral Maxillofac Surg 2005; 43:61-64.

\section{DOI: 10.1038/bdj.2007.414}

\section{News accountability}

Sir, I feel the need to amplify the comments made by Professor Hughes (BDJ 2007; 202: 304) regarding the reports published in the News section of the $B D J$. I have been frustrated by the lack of a complete reference or sometimes any journal reference in these reports. I have found many of the items interesting and obviously newsworthy.

In the same issue, a sentence in a news story was attributed to the Journal of Periodontology whereas a search revealed that it was actually from the American Academy of Periodontology website.

In my view there needs to be a degree of accountability when publishing these news items. The reporting should be accurate if not critical. A full journal reference should be published and ideally the name of the compiler. I believe this format works well in the Journal of the American Dental Association as an example.

\section{E. Gergely}

York

DOI: 10.1038/bdj.2007.415 


\section{Lost in the system}

Sir, having completed a resident post in oral and maxillofacial surgery, and being a non-resident on-call for a maxillofacial team at a Children's Hospital, it became apparent the number of doctors in the Accident and Emergency Department who were unable to correctly read facial radiographic views and diagnose maxillofacial injuries. This was especially noticeable during the months of August and February. This period of time is notorious for mistakes occurring, as Senior House Officers (SHOs) change their rotations, and those that have been in the ActE department for six months and have become adept at diagnosing trauma patients, then rotate onto another department.

This meant that maxillofacial SHOs were unnecessarily called out for injuries that did not require specialist treatment, but, even more worryingly, some patients who had facial injuries were not referred at the appropriate time.

Examples of such misdiagnoses included a case of periorbital ecchymosis and subconjunctival haemorrhage with no radiographs taken at the time. These patients would usually then be referred to the outpatients department, where a correct diagnosis would be made some days later.

There were inevitably some patients who would be incorrectly diagnosed with no follow-up appointment in place, and could simply then get 'lost in the system'. In one of the worst cases, an orbital blow-out fracture came to be diagnosed 10 days post-injury, with signs of diplopia due to the entrapment of the inferior rectus muscle of the affected eye. It has been reported in the literature that one of the commonest fractures missed on radiographs in a one year study of an A\&tE department included those involving the zygoma. ${ }^{1}$

Incorrect terminology was also frequently applied; I once received a telephone referral from an ActE SHO stating with conviction that the "top part of the lower maxilla was fractured on the right side'. Radiographs revealed a fracture of the right condylar head of the mandible, with a second fracture of the left body of the mandible, which had been missed completely.

Another difficulty faced by A\&tE doctors appears to be distinguishing between trauma to the primary and permanent dentitions. I appreciate that oral and maxillofacial surgery is its own speciality, and that we as BDS graduates undergo five years of training to be of a sufficient standard to practise in our chosen field. However, a simple one or two day intensive course may suffice for all new A\&tE doctors, which could be delivered in a similar way as the 'Dentist on the Ward Course' that is offered to us when we embark upon our role as maxillofacial SHOs in a general hospital.

As well as improvements in the teaching of junior doctors, especially in the interpretation of facial radiographic views, increased supervision by senior medical staff may be appropriate, especially in the first few weeks of the new ActE rotations. In the interests of patient safety, and in the increasing climate of medico-legal claims, it is imperative that such mistakes are avoided wherever possible.

\section{H. Dhaliwal}

Birmingham

1. Guly H R. Diagnostic errors in an accident and emergency department. Emerg Med J 2001; 18: 263-269.

\section{DOI: $10.1038 / b d j .2007 .416$}

\section{Drawn into the backwash}

Sir, your editorial A profession no longer (BDJ 2007; 202: 235) is an undisguised anti-government diatribe and I can sympathise, but not altogether agree, with many of the points you make. The medical doctors brought government control upon themselves by failing to set their own targets for the health of the nation, by failing to regulate themselves and failing to instigate changes in structure and management of the NHS to make it fit for purpose in the twenty-first century. My word, how they hate change! The dental profession has, somewhat unfairly, been drawn into the backwash of all this, but in what ways is the new GDC council going to be irretrievably awful compared with that which we have now? You say that without elected dentists on the council 'In future our annual retention fee is just that, a sum of money to allow us the apparent privilege to carry out work in our field' and later 'Perhaps that should now be labelled as another of the stealth taxes - the Licence to Work Tax'.

But surely, that is exactly what the annual retention fee (ARF) is now. It is a flat rate that was imposed by the council in an autocratic authoritarian way. It discriminates against many dentists who are willing to work but not necessarily full-time, because it is propor- tionately greater the lower one's income. It discriminates against women with families who wish to work part-time, it discriminates against young postgraduates on grants, it discriminates against academics for most of their lives because they have low incomes, but worst of all it discriminates against $m e$ because I am over 65 and now work considerably less than full-time. The good work that the GDC did by instigating specialist lists is about to be undermined because it is now going to charge $£ 50$ per annum for each specialty, thereby putting a tax on self improvement for ever. In these days of electronic record keeping, by all means charge a fee for initial entry but thereafter the cost of maintaining a database is minuscule.

So - the ARF is nothing less than a poll tax, imposed by a council having elected dentists. The President himself (a dentist) has explained, with breathtaking sophistry and insouciance, that it is a fee charged for putting dentists' names on a register. Without your entry you cannot practise, there is no room for negotiation, there is no prospect of a sliding scale based on income from dental work. The dentists have had their day and look what we have.

M. Griffiths

Clifton-upon-Teme

DOI: $10.1038 / b d j .2007 .417$

\section{Orthodontic restrictions}

Sir, I wish to express my grave concerns about the restriction on orthodontic treatment on malocclusions in children due to the new NHS dental contract.

Young patients who have been on a waiting list of up to two years now find that when they see the orthodontist, they and their parents are told that they no longer can have their teeth straightened on the NHS. These are children who may have $5 \mathrm{~mm}$ anterior overjets or malaligned upper or lower anterior teeth. These children may as a result of non-treatment suffer early trauma to upper anterior teeth, embarrassment about their appearance and also subject these young people to a lifetime of periodontal treatment when lower anteriors are overcrowded.

Many parents are unable to fund private orthodontic treatment.

I am sending a copy of this letter to the Welsh Assembly member for the area.

P. R. G. Young

Ceredigion

DOI: $10.1038 / b d j .2007 .418$ 